Quim. Nova, Vol. 29, No. 5, 1110-1113, 2006

\title{
DETERMINAÇÃO DOS TEORES DE COBRE E GRAU ALCOÓLICO EM AGUARDENTES DE CANA PRODUZIDAS NO ESTADO DE MINAS GERAIS
}

\author{
Renata Adriana Labanca e Maria Beatriz Abreu Glória* \\ Departamento de Alimentos, Faculdade de Farmácia, Universidade Federal de Minas Gerais, Av. Antonio Carlos, 6627, \\ 31270-901 Belo Horizonte - MG, Brasil \\ Vitor José Pinto Gouveia \\ Centro Tecnológico de Minas Gerais, Av. José Candido da Silveira, 2000, 31170-000 Belo Horizonte - MG, Brasil \\ Robson José de Cássia Franco Afonso \\ Departamento de Química, Universidade Federal de Ouro Preto, R. Diogo de Vasconcelos, 122, \\ 35400-000 Ouro Preto - MG, Brasil
}

Recebido em 1/8/05; aceito em 17/10/05; publicado na web em 14/6/06

\begin{abstract}
DETERMINATION OF COPPER AND ALCOHOL CONTENTS IN SUGAR CANE SPIRITS PRODUCED IN THE STATE OF MINAS GERAIS, BRAZIL. The objective of this work was to investigate if producers of sugar cane spirits in Minas Gerais, Brazil, have improved the copper content of their products and also if they have adjusted to the new standards of identity for 'cachaça' and 'aguardente'. Seventy-one samples, obtained from May 2003 until March 2004, were analyzed. Mean copper content was 2.30 $\mathrm{mg} / \mathrm{L}$, which indicates a significant reduction in levels. The mean alcohol content was $45.6 \% \mathrm{v} / \mathrm{v}$. All of the 'aguardente' but only $79 \%$ of the 'cachaça' attended to the standard of identity for alcohol content for these products.
\end{abstract}

Keywords: sugar cane spirit; copper; alcohol content.

\section{INTRODUÇÃO}

O Brasil destaca-se na produção de aguardente de cana-de-açúcar, sendo esta a segunda bebida alcoólica mais consumida no país. De acordo com o Decreto no. 4851 de $2003^{1}$ e com a Instrução Normativa no. 13 de 20 de junho de $2005^{2}$, aguardente de cana é a bebida com graduação alcoólica de 38 a $54 \%$ v/v à $20{ }^{\circ} \mathrm{C}$, obtida do destilado alcoólico simples de cana-de-açúcar ou pela destilação do mosto fermentado do caldo de cana-de-açúcar, podendo ser adicionada de açúcares até $6 \mathrm{~g} / \mathrm{L}$, expressos em sacarose. A cachaça, entretanto, foi definida como sendo a denominação típica e exclusiva da aguardente de cana produzida no Brasil, com graduação alcoólica de 38 a $48 \%$ v/v à $20{ }^{\circ} \mathrm{C}$, obtida pela destilação do mosto fermentado do caldo de cana-de-açúcar com características sensoriais peculiares, podendo ser adicionada de açúcares até $6 \mathrm{~g} / \mathrm{L}$, expressos em sacarose $\mathrm{e}^{1,2}$.

A produção anual de aguardente de cana no Brasil é de 1,3 bilhões de L. O grande desafio dos produtores tem sido agregar valor ao produto, obter reconhecimento internacional como produto brasileiro e aumentar as exportações ${ }^{3,4}$. Minas Gerais é o quinto produtor nacional de aguardente industrial, porém o estado se destaca na produção por pequenos e médios produtores, que utilizam alambiques de cobre, com um total anual de 120 milhões de L, gerando cerca de 120 mil empregos diretos e três vezes mais empregos indiretos nos setores que gravitam em torno dela durante a entressafra agrícola 5 .

A aguardente produzida em alambique de cobre apresenta melhor qualidade sensorial quando comparada à produção em alambique confeccionado com outros materiais, como aço inox, alumínio e porcelana. A utilização de equipamentos de cobre nos alambiques é favorável por reduzir a acidez e os níveis de aldeídos e compostos sulfurosos, os quais conferem à bebida, sabor e odor

*e-mail: beatriz@farmacia.ufmg.br estranhos ${ }^{3}$. Porém, o cobre pode contaminar o produto quando o manejo (principalmente a higiene) da produção for inadequado ${ }^{5}$.

O cobre é um nutriente essencial para o funcionamento adequado de vários sistemas enzimáticos importantes. Entretanto, a presença de elevadas concentrações de cobre na aguardente é indesejável, pois é prejudicial à saúde humana ${ }^{6}$. A mais conhecida enfermidade decorrente do acúmulo de cobre nos tecidos é a doença de Wilson, que é um transtorno congênito que se transmite por herança autossômica recessiva, associada a um defeito no transporte de cobre, com diminuição de ceruloplasmina, provocando um acúmulo patológico de cobre, principalmente no fígado e cérebro $^{7-10}$. De acordo com Waggoner et al. ${ }^{11}$, o cobre também está associado a doenças neuro-degenerativas, como a esclerose e as doenças de Menkes e de Alzheimer. Assim sendo, o cobre assume grande importância na qualidade final da aguardente, sendo permitida uma quantidade máxima de $5 \mathrm{mg} / \mathrm{L}$ de acordo com a legislação nacional ${ }^{12}$. Por outro lado, a legislação de outros países não tolera mais que $2 \mathrm{mg} / \mathrm{L}$ de cobre nos destilados alcoólicos ${ }^{3}$.

Além do efeito adverso direto à saúde humana, estudos têm indicado que o cobre está associado à formação de uma substância potencialmente cancerígena, o carbamato de etila, em aguardente. De acordo com Aresta et al..$^{13}$, o principal precursor do carbamato de etila em bebidas destiladas é o íon cianeto, sendo o cobre um dos fatores que afetam a formação. Segundo estes autores, a complexação do $\mathrm{Cu}^{\mathrm{II}}$ ao cianato, formado pela oxidação do cianeto, torna-o mais susceptível ao ataque nucleofílico do etanol, formando o carbamato de etila. Assim sendo, os teores de cobre devem ser minimizados na aguardente de cana para prevenir a formação destas substâncias.

Alguns pesquisadores têm se preocupado com os teores de cobre em aguardentes. Lima Neto et al. ${ }^{14}$ encontraram teores de não detectado (nd) a 14,3 mg/L em 73 amostras brasileiras. Silva et al. ${ }^{15}$ detectaram teores de nd a $12,5 \mathrm{mg} / \mathrm{L}$ em 7 amostras de Piracicaba, SP. Em Minas Gerais, Vargas e Glória ${ }^{16}$ avaliaram a qualidade de 511 amostras de aguardente de cana produzidas, comercializadas e/ou engarrafadas nesse estado no período de 1989 
a 1992. Um terço das amostras analisadas $(32,9 \%)$ não atenderam à legislação vigente, tendo sido encontrado um teor máximo de $26 \mathrm{mg} / \mathrm{L}$. Azevedo et al..$^{5}$ analisaram 45 amostras de aguardentes produzidas no período de agosto de 2000 a julho de 2001 no norte e sul de Minas Gerais. Estes autores observaram uma melhoria significativa na qualidade da bebida: apenas $7 \%$ das amostras não atenderam à legislação, sendo o teor máximo encontrado de $12,2 \mathrm{mg} / \mathrm{L}$. A melhoria da qualidade das aguardentes, atingindo-se $100 \%$ de aprovação, é necessária para garantir a saúde da população e disponibilizar um produto com maior aceitação e competitividade no mercado internacional.

O método oficialmente aceito no Brasil para determinação de cobre em aguardente é a quantificação espectrofotométrica com dietilditiocarbamato em álcool amílico ${ }^{17}$. A "Association of Official Analytical Chemists (AOAC)" recomenda, para determinação do cobre em bebidas destiladas, a espectrometria de absorção atômica em chama, com os padrões preparados em solução $50 \%$ v/v de etanol e água ${ }^{18}$. Este método apresenta a vantagem de possuir menor limite de detecção, ser mais rápido e aceito ${ }^{19}$ internacionalmente.

Este trabalho teve como objetivo verificar se a qualidade das aguardentes produzidas no estado de Minas Gerais continua evoluindo com relação aos teores de cobre, utilizando para tal uma metodologia mais sensível ${ }^{18}$. O teor alcoólico também foi determinado para verificar a adequação das amostras ao padrão de identidade e qualidade de cachaça imposto recentemente pela legislação ${ }^{1,2}$ em 38 a $48 \%$ v/v.

\section{PARTE EXPERIMENTAL}

\section{Amostras}

Amostras de cachaça e de aguardente de cana, em um total de 71 , foram coletadas aleatoriamente no mercado consumidor de Belo Horizonte, no período de maio de 2003 a março de 2004. As amostras foram submetidas às análises de grau alcoólico e teores de cobre. Os resultados foram analisados individualmente e agrupados em quatro regiões - Metropolitana, Noroeste, Sudoeste e Leste, sendo que as duas últimas englobam algumas macrorregiões propostas pelo $\mathrm{IBGE}^{20}$. A região Sudoeste inclui as macrorregiões Sul de Minas, Alto São Francisco e Triângulo Mineiro e a Leste engloba as macrorregiões Vale do Jequitinhonha, Zona da Mata e Rio Doce.

\section{Reagentes e padrões}

A maioria dos reagentes utilizados era de grau analítico. A curva padrão foi feita utilizando soluções de cobre titrisol nas concentrações de 0,$50 ; 1,00 ; 2,00$; e 4,00 mg/L em etanol 50\% ${ }^{17,18}$. As amostras foram analisadas diretamente. As amostras cujas leituras extrapolaram a curva padrão foram diluídas com etanol 50\% e submetidas novamente à análise. A curva de calibração foi construída e verificada a linearidade da resposta.

\section{Determinação do grau alcoólico}

O grau alcoólico foi determinado pelo método de densitometria em picnômetro de vidro, com capacidade para $50 \mathrm{~mL}$, segundo metodologia da $\mathrm{AOAC}^{18}$ de número 942.06. Os picnômetros foram calibrados com água destilada e os dados coletados em triplicata. As análises foram feitas a $20{ }^{\circ} \mathrm{C}$. A partir da densidade determinou-se o grau alcoólico expresso em \% v/v, por meio de tabela de conversão ${ }^{12}$.

\section{Determinação do teor de cobre}

Os teores de cobre foram determinados por leitura direta utilizando espectrômetro de absorção atômica, segundo metodologia descrita pela $\mathrm{AOAC}^{18}$. As determinações foram feitas em espectrômetro de absorção atômica (Perkin-Elmer - modelo 5000, Alemanha), com chama de ar e acetileno nos fluxos de 4,0 e 2,0 L/ min, respectivamente, queimador do tipo monofenda e comprimento de onda de $324,8 \mathrm{~nm}$

\section{Análise estatística}

Todas as análises foram feitas em triplicata. Os resultados obtidos foram agrupados por região de origem e submetidos à análise de variância, sendo as médias comparadas pelo teste de Duncan a $5 \%$ de probabilidade.

\section{RESULTADOS E DISCUSSÃO}

\section{Grau alcoólico}

Os valores obtidos para o grau alcoólico variaram de 29,2 a $57,4 \%$ v/v (Figura 1), sendo o teor médio e a mediana de $45,6 \%$ v/v e o desvio padrão de $3,7 \%$ v/v, o que corresponde a um coeficiente de variação de $9,5 \%$. Ao analisar os rótulos das aguardentes, observou-se que das 71 amostras, 8 foram denominadas aguardente e as demais, cachaça. As amostras de aguardente apresentaram grau alcoólico que variou de 41,0 a 48,9\% v/v (média de 45,9\% v/v), o que está coerente com o Decreto 4851 de outubro de $2003^{1}$ e com a Instrução Normativa no. 13 de 29 de junho de $2005^{2}$, que diferenciam cachaça de aguardente. Por outro lado, dentre as 63 amostras de cachaça analisadas, $13(21 \%)$ estavam com teores de álcool acima de $48 \%$ v/v e 2 amostras (3\%) estavam abaixo de $38 \% \mathrm{v} / \mathrm{v}$. Desta forma, das 71 amostras analisadas, apenas 79\% atenderam à legislação vigente quanto a este parâmetro.

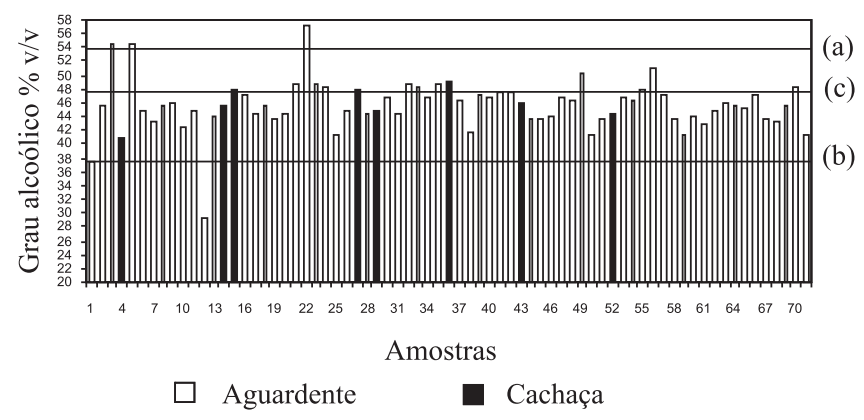

Figura 1. Grau alcoólico em amostras de aguardentes disponíveis no mercado consumidor de Belo Horizonte, MG, no período de maio de 2003 a março de 2004. $n=3$; Limites para aguardente: (a) máximo $=54 \%$ v/v e (b) mínimo $=$ $38 \% \mathrm{v} / \mathrm{v}$. Limites para cachaça: (c) máximo $=48 \% \mathrm{v} / \mathrm{v}, e(b)$ mínimo $=38 \% \mathrm{v} / \mathrm{v}$

Vargas e Glória ${ }^{16}$ e Azevedo et al..$^{5}$ observaram um maior percentual de atendimento à legislação em amostras analisadas no período de 1989 a 1992 - média de $98 \%$ e no período de agosto de 2000 a julho de 2001 - média de 93\%. A diminuição significativa no percentual de atendimento à legislação observada neste estudo indica que os produtores ainda não se adequaram ao padrão de identidade e qualidade da cachaça. Os resultados obtidos neste estudo foram mais amplos que aqueles observados por Azevedo et $a l .{ }^{5}$, que encontraram valores de 34 a $50 \%$ v/v para amostras de Minas Gerais. 
Ao agrupar os resultados em função da região geográfica, as amostras não apresentaram diferenças significativas quanto ao grau alcoólico (Tabela 1). Com relação ao percentual de atendimento à legislação das amostras de aguardente analisadas em função da região (Figura 2), observa-se que a Leste foi a que apresentou maior percentual de amostras que não atenderam a legislação.

Tabela 1. Grau alcoólico encontrado em aguardentes produzidas em diferentes regiões de Minas Gerais, no período de maio de 2003 a março de 2004

\begin{tabular}{lccc}
\hline Macrorregiões & $\mathrm{n}$ & \multicolumn{2}{c}{ Grau alcoólico $(\% \mathrm{v} / \mathrm{v})^{*}$} \\
& & faixa & média $\pm \mathrm{dp}$ \\
\hline Metropolitana & 19 & $41,3-48,7$ & $45,6 \pm 2,4$ \\
Noroeste & 26 & $29,2-48,7$ & $44,5 \pm 3,7$ \\
Sudoeste & 13 & $37,5-57,4$ & $46,9 \pm 5,8$ \\
Leste & 13 & $43,8-51,0$ & $46,6 \pm 2,4$ \\
Total & 71 & $29,2-57,4$ & $45,6 \pm 3,7$ \\
\hline
\end{tabular}

* Valores médios ( \pm desvio padrão) não diferem significativamente pelo teste de Duncan a $5 \%$ de probabilidade.

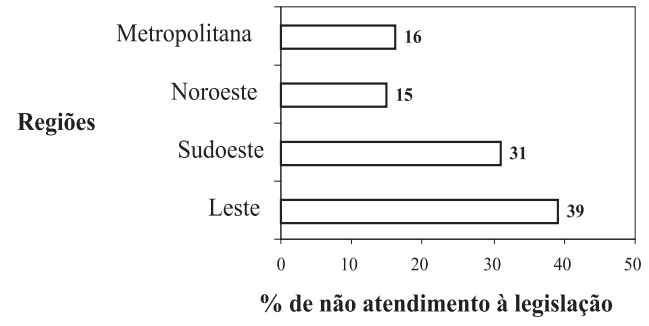

Figura 2. Percentual de amostras de aguardente de cana produzidas em diferentes regiões de Minas Gerais, no período de maio de 2003 a março de 2004, que não atenderam à legislação vigente com relação ao grau alcoólico

\section{Teor de cobre}

O cobre foi detectado em todas as amostras de aguardente analisadas em teores que variaram de 0,05 a $8,10 \mathrm{mg} / \mathrm{L}$, sendo o teor médio de 2,30 mg/L (Figura 3). Sete por cento das amostras não atenderam à legislação vigente ${ }^{12}$, que limita o teor de cobre a um valor máximo de $5 \mathrm{mg} / \mathrm{L}$.

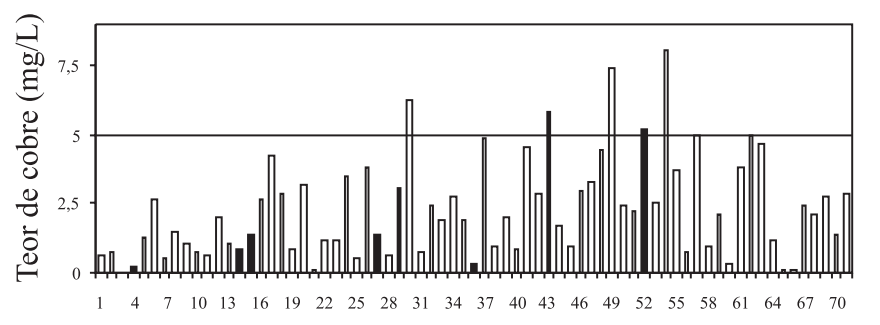

$$
\text { Amostras } \square \text { Cachaça }
$$

Figura 3. Teor de cobre em amostras de aguardentes disponíveis no mercado consumidor de Belo Horizonte, MG, no período de maio de 2003 a março de 2004 (limite máximo permitido pela legislação vigente $=5,0 \mathrm{mg} / \mathrm{L}$ )

Estes resultados são semelhantes aos encontrados por Azevedo et al..$^{5}$ que, em 45 amostras de aguardente de Minas Gerais, encontraram $7 \%$ com teores de cobre acima do permitido. Porém, os percentuais encontrados por Küchler e $\operatorname{Silva}^{21}$ ao avaliarem 21 amostras de aguardente de diferentes regiões do Brasil foram maiores, ou seja, $14 \%$ em desacordo com a legislação quanto à presença de cobre. Nascimento et al. ${ }^{22}$ encontraram teor médio de cobre abaixo de $5 \mathrm{mg} / \mathrm{L}$ em 69 amostras de aguardente analisadas, entretanto, a faixa de resultados foi mais ampla, variando de não detectado $(<0,1 \mathrm{mg} / \mathrm{L})$ a $14,3 \mathrm{mg} / \mathrm{L}$.

Ao se agrupar as amostras por região, observa-se diferença nos teores de cobre a $5 \%$ de probabilidade (Tabela 2). Os menores teores de cobre foram detectados em amostras da região Metropolitana, na qual nenhuma amostra apresentou teor de cobre acima do estabelecido pela legislação vigente. Não foi observada diferença significativa nos teores médios de cobre para as demais regiões.

Tabela 2. Teores de cobre encontrados em aguardentes produzidas em diferentes regiões de Minas Gerais, no período de maio de 2003 a março de 2004

\begin{tabular}{|c|c|c|c|}
\hline \multirow[t]{2}{*}{ Regiões } & \multirow[t]{2}{*}{$\mathrm{n}$} & \multicolumn{2}{|c|}{ Teor de cobre $\mathrm{mg} / \mathrm{L}^{*}$} \\
\hline & & faixa & média \pm dp \\
\hline Metropolitana & 19 & $0,05-4,25$ & $1,37 \pm 1,07 \mathrm{~b}$ \\
\hline Noroeste & 26 & $0,10-6,25$ & $2,28 \pm 1,70 \mathrm{a}$ \\
\hline Sudoeste & 13 & $0,10-7,40$ & $2,57 \pm 1,90 \mathrm{a}$ \\
\hline Leste & 13 & $0,40-8,10$ & $3,42 \pm 2,20 \mathrm{a}$ \\
\hline Total & 71 & $0,05-8,10$ & $2,30 \pm 1,81$ \\
\hline
\end{tabular}

*Valores médios ( \pm desvio padrão) com uma mesma letra não diferem significativamente pelo teste de Duncan a 5\% de probabilidade.

A principal justificativa para altos teores de cobre em aguardentes pode estar relacionada à falta de higiene dos alambiques, sendo o cobre proveniente da dissolução do "azinhavre" (carbonato básico de cobre solúvel em ácido) que se forma no interior do alambique e, principalmente, nas partes internas da serpentina da resfriadeira. Com assepsia adequada do aparelho, pode-se obter aguardente com um teor de cobre menor ${ }^{5,23}$. Cardoso ${ }^{24}$ recomenda que a primeira destilação do dia deve ser feita adicionando-se suco de limão (ou ácido cítrico) à água na concentração de 5 L do suco para 100 L de água. Assim, esta removerá o azinhavre existente no interior da serpentina de condensação dos vapores do alambique. Segundo Bizelli et $a l .^{23}$, a bidestilação das aguardentes pode também ser utilizada, permitindo a redução dos teores de cobre. A contaminação da aguardente por cobre também pode ser reduzida utilizando-se carvão ativo ou resinas de troca iônica. Entretanto, os compostos que dão o sabor e aroma característicos das aguardentes podem ser igualmente retidos, prejudicando assim a qualidade do produto final ${ }^{5,22}$.

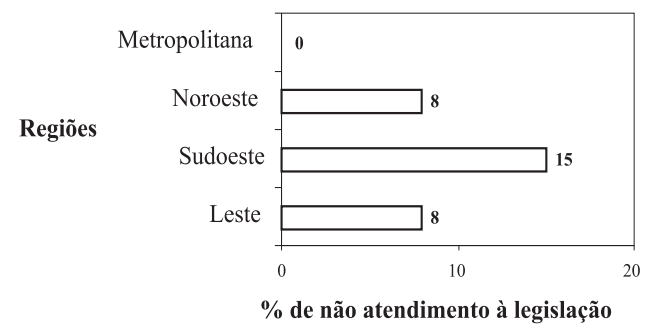

Figura 4. Percentual de amostras de aguardente de cana produzidas em diferentes regiões de Minas Gerais, no período de maio de 2003 a março de 2004, que não atenderam à legislação vigente com relação ao cobre

Vargas e Glória ${ }^{16}$ avaliaram os teores de cobre em amostras de aguardente de cana produzidas ou engarrafadas em Minas Gerais 
nos anos de 1989, 1990, 1991, 1992, 1993 e 1994, encontrando percentuais de atendimento que aumentaram ao longo dos anos de $49,68,75,72,80$ e 82 , respectivamente. Os resultados obtidos neste estudo (93\% de atendimento) indicam que os produtores têm envidado esforços para melhorar a qualidade de seus produtos. Além disto, o teor médio obtido neste estudo $(2,3 \mathrm{mg} / \mathrm{L})$ foi menor se comparado ao obtido por Vargas e Glória ${ }^{16}$, cujo valor era de 4,5 mg/L.

Apesar da maioria dos produtores de aguardente não encontrarem barreira sanitária para o cobre no mercado interno, o mesmo não ocorreria quando se tratar de mercado internacional. Dentre as 71 amostras analisadas, apenas 50,7\% atenderiam ao limite de $2 \mathrm{mg} / \mathrm{L}$ de cobre nos destilados alcoólicos imposto pelo mercado internacional. Entretanto, levando-se em consideração o papel do cobre na formação de carbamato de etila, deve-se procurar minimizar os teores de cobre em aguardentes.

Estudo de correlação feito entre os teores de cobre encontrados e o grau alcoólico indicou não haver correlação significativa entre estes parâmetros para as amostras analisadas.

\section{CONCLUSÕES}

As aguardentes de cana produzidas em Minas Gerais apresentaram grau alcoólico de 29,2 a $57,4 \%$ v/v e teores de cobre de 0,05 a $8,10 \mathrm{mg} / \mathrm{L}$. Não houve diferença significativa nos valores médios obtidos para diferentes regiões do estado com relação ao grau alcoólico, entretanto, menores teores de cobre foram obtidos para a região metropolitana.

Houve uma melhoria significativa na qualidade das aguardentes de cana mineiras com relação aos teores de cobre na última década. Entretanto, os teores encontrados continuariam sendo barreiras a nível internacional para 49,3\% dos produtores. Entretanto, com relação ao grau alcoólico, houve um aumento no percentual de não atendimento à legislação, o que indica que os produtores ainda não se adequaram à legislação que diferencia cachaça de aguardente.

\section{REFERÊNCIAS}

1. Brasil. Decreto no. 4851 de 02 de outubro de 2003.

2. Brasil. Instrução Normativa no. 13, de 29 de junho de 2005, Diário Oficial da União, s.1, p. 3, Brasília, 30/06/2005.

3. Cardoso, D. R.; Lima-Neto, B. S.; Franco, D. W.; Nascimento, R. F.; Quim. Nova 2003, 26, 165

4. http://www.udop.com.br/diversas/materias/ div_29_07_08.htm, acessada em Março 2003.

5. Azevedo, S. M.; Cardoso, M. G.; Pereira, N. E.; Ribeiro, C. F. S.; Silva, V. F.; Aguiar, F. C.; Ciênc. Agrotecnol. 2003, 27, 618.

6. Mosha, D.; Wangabo, J.; Mhinzi, G.; Food Chem. 1996, 57, 205.

7. Campos-Franco, J.; Dominguez-Santalla, M. J.; Tomé-Martinez De Rituerto, S.; Otero-Antón, E.; González-Quintela, A.; An. Med. Intern. 2003, 20, 416 .

8. Gow, P. J.; Smallwood, R. A.; Angus, P. W.; Smith, A. L.; Wall, A. J.; Sewell, R. B.; Gut 2000, 46, 415.

9. Loudianos, G.; Gitlin, J. D.; Semin. Liver Dis. 2000, 20, 353.

10. Sargentelli, V.; Mauro, A. E.; Massabni, A. C.; Quim. Nova 1996, 19, 290.

11. Waggoner, D. J.; Bartnikas, T. B.; Gittlin, J. D.; Neurobiol. Dis. 1999, 6, 221.

12. Brasil. Portaria n ${ }^{\circ}$ 371, MARA. Diário Oficial da União, s. 1, p. I, Supl. Brasília, 19/09/1974.

13. Aresta, M.; Boscolo, M.; Franco, D. W.; J. Agric. Food Chem. 2001, 49, 2819.

14. Lima Neto, B. S.; Bezerra, C. W. B.; Polastro, L. R.; Campos, P.; Nascimento, R. F.; Furuya, S. M. B.; Franco, D. W.; Quim. Nova 1994, $17,220$.

15. Silva, R. M. C.; Almeida, E.; Valencia, E. P. E.; Nascimento Filho, V. F.; J. Radioanal. Nucl. Chem. 2004, 260, 3.

16. Vargas, E. A.; Glória, M. B. A.; Ciênc. Tecnol. Aliment. 1995, 15, 43.

17. Instituto Adolfo Lutz; Normas Analíticas do Instituto Adolfo Lutz, São Paulo, 1985, vol. 1.

18. Association of Official Analytical Chemists; Official Methods of Analysis, Arlington: AOAC, 1995.

19. Honorato, F. A.; Honorato, R. S.; Pimentel, M. F.; Araujo, M. U.; The Analyst 2002, 127, 1520.

20. http://www.ibge.gov.br, acessada em Março 2003.

21. Küchler, I. L.; Silva, F. A. M.; Quim. Nova 1999, 22, 339.

22. Nascimento, R. F.; Bezerra, C. W. B.; Furuya, S. M. B.; Schultz, M. S.; Polastro, L. S.; Lima B. S.; Franco, D. W.; J. Food Compos. Anal. 1999, $12,17$.

23. Bizelli, L. C.; Ribeiro, C. A. F.; Novaes, F. V.; Sci. Agricol. 2000, 57, 100.

24. Cardoso, M. G.; Produção artesanal de aguardente, UFLA: Lavras, 2000. 